\title{
Early vaginal progesterone versus Placebo in Twin Pregnancies for prevention of spontaneous preterm birth (EVENTS): A randomised double-blind trial
}

Anoop Rehal, M.D., ${ }^{1}$ Zsófia Benkő, M.D., ${ }^{1}$ Catalina De Paco Matallana, M.D., ${ }^{2}$ Argyro Syngelaki, Ph.D., ${ }^{1}$ Deepa Janga, M.D., ${ }^{3}$ Simona Cicero, M.D., ${ }^{4}$ Ranjit Akolekar, M.D., ${ }^{5}$ Mandeep Singh, M.D. ${ }^{6}$ Petya Chaveeva, M.D., ${ }^{7}$ Jorge Burgos, M.D., ${ }^{8}$ Francisca S Molina, M.D., ${ }^{9}$ Makrina Savvidou, M.D., ${ }^{10}$ Maria De La Calle, M.D. ${ }^{11}$ Nicola Persico, M.D., ${ }^{12}$ Maria Soledad Quezada Rojas, M.D., ${ }^{13}$ Ashis Sau, M.D. ${ }^{14}$ Elena Greco, M.D.,${ }^{15}$ Neil O'Gorman, M.D. ${ }^{16}$ Walter Plasencia, M.D., ${ }^{17}$ Susana Pereira, M.D.,${ }^{18}$ Jacques C Jani, M.D., ${ }^{19}$ Nuria Valino, M.D. ${ }^{20}$ Maria del Mar Gil, M.D., ${ }^{21}$ Kate Maclagan, Ph.D., ${ }^{22}$ Alan Wright, Ph.D., ${ }^{23}$ David Wright, Ph.D., ${ }^{23}$ Kypros H Nicolaides M.D. ${ }^{1}$

1. Fetal Medicine Research Institute, King's College Hospital, London, UK

2. Hospital Clínico Universitario Virgen de la Arrixaca and Institute for Biomedical Research of Murcia, IMIB-Arrixaca, Murcia, Spain

3. North Middlesex University Hospital, London, UK

4. Homerton University Hospital, London, UK

5. Medway Maritime Hospital, Gillingham, UK

6. Southend University Hospital, Westcliff-on-Sea, UK

7. Dr. Shterev Hospital, Sofia, Bulgaria

8. Hospital Universitario Cruces, Biocruces Bizkaia Health Research Institute, UPV/EHU, Bizkaia, Spain

9. Hospital Universitario San Cecilio, Instituto de Investigación Biosanitaria (IBS) Granada, Spain

10. Chelsea and Westminster Hospital, Imperial College London, UK

11. Hospital Universitario La Paz, Madrid, Spain

12. Ospedale Maggiore Policlinico, Milan and Department of Clinical Sciences and Community Health, University of Milan, Italy

13. Hospital Universitario "12 De Octubre", Madrid, Spain

14. Lewisham University Hospital, London, UK

15. Royal London Hospital, London, UK

16. Hospital Necker Enfants Malades, Paris, France

17. Hospiten Group, Tenerife, Spain

18. Kingston Hospital, Kingston upon Thames, UK

19. University Hospital Brugmann, Université Libre de Bruxelles, Brussels, Belgium

20. University Hospital A Coruña, Spain

21. Hospital Universitario de Torrejón and School of Health Sciences. Universidad Francisco de Vitoria, Madrid, Spain.

22. Fetal Medicine Foundation, London, UK

23. University of Exeter, Exeter, UK

Clinical trial registration: EudraCT number 2015-005180-16; ISRCTN.com, number ISRCTN66445401).

Conflict of interest: None

Sources of Funding: The study was supported by a grant from the Fetal Medicine Foundation (Charity No: 1037116). The placebo and progesterone capsules were manufactured, packaged, labelled, stored and distributed free of charge by Besins Healthcare, Bruxelles, Belgium. These bodies had no involvement in the study design; in the collection, analysis and interpretation of data; in the preparation, review, or approval of the manuscript; and in the decision to submit the article for publication.

\section{Correspondence:}

Professor KH Nicolaides, Fetal Medicine Research Institute,

King's College Hospital, 16-20 Windsor Walk, Denmark Hill, London SE5 8BB

Telephone: +442032998256; email: kypros@fetalmedicine.com

Abstract word count: 677 , Main text word count: 3604 . 


\section{CONDENSATION}

In women with twin pregnancies universal treatment with vaginal progesterone did not reduce the incidence of spontaneous birth between $24^{+0}$ and $33^{+6}$ weeks' gestation.

Short version of article title: Vaginal progesterone in unselected twin pregnancies.

\section{AJOG AT A GLANCE}

- Randomized controlled trial testing the hypothesis that in women with twin pregnancies, vaginal progesterone at a dose of $600 \mathrm{mg}$ per day from 11-14 until 34 weeks' gestation, as compared with placebo, would result in a significant reduction in the incidence of spontaneous preterm birth between $24^{+0}$ and $33^{+6}$ weeks.

- In women with twin pregnancies universal treatment with vaginal progesterone did not reduce the incidence of spontaneous birth between $24+0$ and 33+6 weeks' gestation. Post hoc time to event analysis led to the suggestion that progesterone may reduce the risk of spontaneous birth $<32$ weeks in women with cervical length $<30 \mathrm{~mm}$ and it may increase the risk for those with cervical length $\geq 30 \mathrm{~mm}$.

- In women with twin pregnancies universal treatment with vaginal progesterone does not reduce the incidence of early spontaneous birth. 


\section{ABSTRACT}

Background: In women with a singleton pregnancy and sonographic short cervix in midgestation, vaginal administration of progesterone reduces the risk of early preterm birth and improves neonatal outcomes, without any demonstrable deleterious effects on childhood neurodevelopment. In women with twin pregnancies the rate of spontaneous early preterm birth is 10-times higher than in singletons and in this respect all twins are at increased risk of preterm birth. However, six trials in unselected twin pregnancies reported that vaginal progesterone from mid-gestation had no significant effect on the incidence of early preterm birth. Such apparent lack of effectiveness of progesterone in twins may be due to inadequate dosage or treatment which is started too late in pregnancy.

Objective: The Early vaginal progesterone for the preVention of spontaneous prEterm birth iN TwinS: A randomized, placebo controlled, double-blinded trial (EVENTS) was designed to test the hypothesis that, among women with twin pregnancies, vaginal progesterone at a dose of 600 mg per day from 11-14 until 34 weeks' gestation, as compared with placebo, would result in a significant reduction in the incidence of spontaneous preterm birth between $24^{+0}$ and $33^{+6}$ weeks.

Methods: The trial was conducted at 22 hospitals in England, Spain, Bulgaria, Italy, Belgium and France. Women were randomly assigned in a 1:1 ratio, to receive either progesterone or placebo and in the random-sequence generation there was stratification according to participating center. Primary outcome was spontaneous birth between $24^{+0}$ and $33^{+6}$ weeks' gestation. Statistical analyses were performed on an intention-to-treat basis. Logistic regression analysis was used to determine the significance of difference in incidence of spontaneous birth between $24^{+0}$ and $33^{+6}$ weeks' gestation between the progesterone and placebo groups, adjusting for the effect of participating centre, chrorionicity, parity and method of conception. Prespecified tests of treatment interaction effects with chrorionicity, parity, method of conception, compliance and cervical length at recruitment were performed. A post hoc analysis using mixed effects Cox regression was used for further exploration of the effect of progesterone on preterm birth.

Results: We recruited 1,194 women between May 2017 and April 2019; 21 withdrew consent and 4 were lost to follow up, which left 582 in the progesterone group and 587 in the placebo group. Adherence was good, with reported intake of $\geq 80 \%$ of the required number of capsules in $81.4 \%$ of the participants. After excluding births before 24 weeks and indicated deliveries before 34 weeks, spontaneous birth between $24^{+0}$ and $33^{+6}$ weeks occurred in $10.4 \%(56 / 541)$ participants in the progesterone group and in $8.2 \%(44 / 538)$ in the placebo group (odds ratio in the progesterone group, adjusting for the effect of participating center, chrorionicity, parity and method of conception, $1.35 ; 95 \% \mathrm{Cl} 0.88-2.05 ; p=0.17)$. There was no evidence of interaction between the effects of treatment and chorionicity $(p=0.28)$, parity $(p=0.35)$ method of conception $(p=0.56)$ and adherence $(p=0.34)$; however, there was weak evidence of an interaction with cervical length $(p=0.08)$ suggestive of harm to those with cervical length $\geq 30 \mathrm{~mm}$ (odds ratio $1.61,95 \% \mathrm{Cl} 1.01-2.59$ ) and potential benefit for those with cervical length $<30 \mathrm{~mm}$ (odds ratio $0.56 ; 95 \% \mathrm{Cl} 0.20-1.60)$. There was no evidence of difference between the two treatment groups for stillbirth or neonatal death; neonatal complications; neonatal therapy; and poor fetal growth. In the progesterone group there were $1.4 \%(8 / 582)$ women and $1.9 \%(22 / 1,164)$ fetuses with at least one serious adverse event; the respective numbers for the placebo group were $1.2 \%$ $(7 / 587)$ and $3.2 \%(37 / 1,174)(p=0.80$ and $p=0.06$, respectively). In the post hoc time to event analysis, miscarriage or spontaneous preterm birth between randomization and $31^{+6}$ weeks' gestation was reduced in the progesterone group relative to the placebo group (hazard ratio 0.23 , $95 \% \mathrm{Cl} 0.08-0.69)$.

Conclusions: In women with twin pregnancies universal treatment with vaginal progesterone did not reduce the incidence of spontaneous birth between $24^{+0}$ and $33^{+6}$ weeks' gestation. Post hoc time to event analysis led to the suggestion that progesterone may reduce the risk of 
spontaneous birth $<32$ weeks in women with cervical length $<30 \mathrm{~mm}$ and it may increase the risk for those with cervical length $\geq 30 \mathrm{~mm}$. 


\section{INTRODUCTION}

Preterm birth is the leading cause of neonatal and childhood death and disability and the incidence of these adverse events is particularly marked in early preterm birth before 34 weeks of gestation. ${ }^{1,2}$ In singleton pregnancies the rate of spontaneous early preterm birth is about $1 \%$ and in twin pregnancies the rate is 10 -times higher. ${ }^{3}$ There is strong evidence from randomized trials that in women with a singleton pregnancy and sonographic short cervix in mid-gestation, vaginal administration of progesterone in doses of $90-200 \mathrm{mg}$ daily reduces the risk of early preterm birth and improves neonatal outcomes, without any demonstrable deleterious effects on childhood neurodevelopment. ${ }^{4-7}$ In contrast, six previous trials which recruited between 70 and 675 women with unselected twin pregnancies and used between $90 \mathrm{mg}$ and $400 \mathrm{mg}$ of progesterone daily, reported no significant effect on the incidence of early preterm birth..$^{8-13}$ One individual participant data meta-analysis in women with unselected twin pregnancies reported that vaginal progesterone from mid-gestation was not associated with reduction in the rate of adverse perinatal outcome (RR $0.97 ; 95 \% \mathrm{Cl} 0.77-1.2)$, but in a subgroup of women with a cervical length of $\leq 25 \mathrm{~mm}$ at randomization, progesterone reduced the rate of adverse perinatal outcome (RR 0.57; 95\% Cl 0.47-0.70). ${ }^{14}$

The apparent lack of effectiveness of progesterone in twins may be due to inadequate dosage or treatment which is started too late in pregnancy. The Early vaginal progesterone for the preVention of spontaneous prEterm birth iN TwinS: A randomized, placebo controlled, doubleblinded trial (EVENTS) was designed to test the hypothesis that, among women with twin pregnancies, vaginal progesterone at a dose of $600 \mathrm{mg}$ per day from 11-14 until 34 weeks' gestation, as compared with placebo, would result in a significant reduction in the incidence of spontaneous preterm birth before 34 weeks' gestation.

\section{METHODS}

\section{Study design}

This was a double-blind, placebo-controlled trial comparing vaginal progesterone at a dose of $300 \mathrm{mg}$ twice per day with placebo from 11-14 until 34 weeks' gestation in women with twin pregnancies. We conducted the trial at 22 maternity hospitals in England, Spain, Bulgaria, Italy, Belgium and France. All women with twin pregnancies with a routine prenatal visit at $11^{+0}$ to $13^{+6}$ weeks' gestation in the participating hospitals were assessed for eligibility and were offered to participate in this study. At this visit, first, maternal characteristics, medical history and obstetric history were recorded, second, maternal weight and height were measured, third, gestational age was determined from the measurement of the fetal crown-rump length of the larger fetus, and fourth, transvaginal sonography was carried out to determine the cervical length. ${ }^{15}$ Quality control of screening and verification of adherence to protocol were performed by the Fundación para la Formación e Investigación Sanitaria (FFIS) for the sites in Spain and by the Fetal Medicine Foundation for sites in the UK, Bulgaria, Belgium, France and Italy. Approval for the study was obtained in each country where the trial was conducted from the relevant Research Ethics Committee and competent authority.

\section{Participants}

Inclusion criteria for the trial were: age older than 18 years; dichorionic or monochorionic diamniotic twin pregnancy; two live fetuses at the 11-13 weeks' scan; fluent in the local language. Exclusion criteria were: monoamniotic pregnancies, monochorionic diamniotic pregnancies with early signs of twin-to-twin transfusion syndrome, defined as $>20 \%$ discordance in crown-rump length at the 11-13 weeks' scan; major fetal abnormality or nuchal translucency thickness $>3.5$ $\mathrm{mm}$ identified at the 11-13 weeks' scan; women who were unconscious or severely ill, those with learning difficulties or serious mental illness; hypersensitivity to progesterone; regular treatment with progesterone within the previous 7 days; severe hepatic dysfunction; mammary or genital 
tract carcinoma, thrombophlebitis or thromboembolic disorders; porphyria; cerebral hemorrhage; allergy to sunflower oil, soya lecithin, gelatin, glycerol (E422), titanium dioxide (E171); and participation in another drug trial within 28 days. Potential trial participants were given written information about the trial and those who agreed to participate provided written informed consent.

\section{Randomization and masking}

Eligible women were randomly assigned, in a 1:1 ratio, with the use of a simple permuted block provided by Besins Healthcare, Bruxelles, Belgium, to receive either progesterone or placebo and in the random-sequence generation there was stratification according to participating center. The placebo and progesterone capsules were manufactured, packaged, labelled, stored and distributed by Besins Healthcare, Bruxelles, Belgium. The placebo capsules were identical to those of the progesterone in parameters such as size, thickness, physical properties and appearance. Participants, investigators, pharmacists, and others involved in giving the intervention, assessing outcomes, or analyzing data remained masked to treatment allocation until the end of the study.

\section{Procedures}

After randomization, study participants were prescribed the investigational medicinal product, received instructions on self-administration of one vaginal capsule twice daily throughout the study and to stop vaginal insertion of capsules at 34 weeks' gestation or in the event of earlier delivery. Compliance and adverse events were assessed and recorded at follow up clinical visits at 20-22, 24-26, 28-30, 31-33 and 35-37 weeks' gestation in dichorionic twin pregnancies and at 16-17 weeks and every two weeks thereafter in monochorionic twin pregnancies, and in one telephone interview 30 days after the last capsule was taken. Participants were encouraged to record any side effects or adverse events in a diary that was reviewed at each trial visit and they were specifically asked about such events at the telephone interview. We assessed adherence by researchers counting the capsules returned by participants at each visit and by the participants themselves at the telephone interview. The total number of capsules taken was calculated by subtracting the number of capsules returned from the number of capsules prescribed.

\section{Outcome measures}

The primary outcome measure was spontaneous birth between $24^{+0}$ and $33^{+6}$ weeks' gestation inclusive. In cases in which one fetus died (termination, miscarriage or stillbirth) at a gestational age that is earlier than that of the birth of the second fetus, the gestational age for pregnancy outcome was the one at the birth of the second twin.

Secondary outcomes were spontaneous birth between 24 weeks and $<28,<30,<32$ and $<37$ weeks; spontaneous or indicated birth between 24 weeks and $<28,<30,<32,<34$ and $<37$ weeks; spontaneous or indicated birth between randomization and $<24,<28,<30,<32$ and $<34$ weeks; stillbirth or neonatal death; neonatal complications; neonatal therapy; and poor fetal growth (birth weight below $1500 \mathrm{~g}, 2000 \mathrm{~g}$ and below the 3rd, 5th, or 10th percentile). ${ }^{16}$

Adherence was considered to be good if the reported use of capsules was $\geq 80 \%$ of the total number participants should have used between the date of randomization and the date of the 34 weeks visit or delivery if this occurred prior to 34 weeks.

\section{Statistical analysis}

It was hypothesized that vaginal progesterone would reduce the rate of spontaneous birth between $24^{+0}$ and $33^{+6}$ weeks of gestation by $40 \%$, from $13 \%$ in the placebo group to $7.8 \%$ in the progesterone group. We calculated that enrolment of 1,080 participants would give the study a 
power of $80 \%$ to show a treatment effect at a two-sided alpha level of $5 \%$. The target recruitment figure was inflated to 1,188 to account for approximately $10 \%$ attrition.

Statistical analyses were carried out on an intention-to-treat basis, and no interim analyses were performed. Logistic regression analysis was used to determine the significance of difference in incidence of spontaneous birth between $24^{+0}$ and $33^{+6}$ weeks' gestation between the progesterone and placebo groups, adjusting for the effect of participating center, chrorionicity (monochorionic, dichorionic), parity (nulliparous, parous with previous preterm birth, parous without previous preterm birth) and method of conception (in vitro fertilization, natural conception or use of ovulation drugs). The treatment effect was quantified as odds ratio with $95 \%$ confidence interval in the progesterone group. We also produced Kaplan-Meier survival estimates of the cumulative incidence of spontaneous birth between $24^{+0}$ and $33^{+6}$ weeks' gestation according to trial group, in which births before 24 weeks and indicated births before 34 weeks were treated as censored observations. Cox regression was used to test the effect of treatment adjusting for parity, chorionicity and conception.

Prespecified tests of treatment interaction effects with chrorionicity, parity, method of conception, compliance and cervical length at recruitment were performed. Cervical length was included as a continuous covariate defined as $35 \mathrm{~mm}$ minus cervical length for cervical length $<35 \mathrm{~mm}$ and 0 for cervical length $\geq 35 \mathrm{~mm}$. This choice of transformation was made on the basis of analysis of data blinded to treatment allocation. To aid interpretation, analyses were performed to examine the effect of treatment by subgroups defined in terms of chrorionicity, parity, method of conception, compliance and cervical length. The findings from the planned analyses led to a post hoc analysis of gestational age at delivery using mixed effects Cox regression models. The proportional hazards assumption for the treatment effect was clearly inappropriate; the cumulative incidence curves for progesterone and placebo crossed (see results). This led to stratification according to gestational age at delivery. We chose to estimate hazard ratios with stratification of gestational age into two strata: deliveries before 32 weeks and deliveries between $32^{+0}$ and $33^{+6}$ weeks.

Secondary outcomes were compared across treatment groups using mixed effects logistic regression with fixed effects for treatment, parity and chorionicity, and random effects for center. Results were presented as forest plots showing estimates and $95 \%$ confidence intervals (Cl) for treatment effects. Results on perinatal and neonatal outcome were examined both at the pregnancy and fetal / neonatal level. For fetal / neonatal outcomes random effects were included for pregnancy to account for associations between fetuses / neonates of the same mother.

The statistical software package $\mathrm{R}$ was used for data analyses. ${ }^{17-21}$

\section{RESULTS}

\section{Trial participants}

Recruitment to the trial started in May 2017 and was completed in April 2019. A total of 2,456 women with twin pregnancies were screened and $523(21.3 \%)$ of these were excluded from recruitment to the trial because they did not fulfil the eligibility criteria (Fig. 1). Of the 1,933 eligible women, $1,194(61.8 \%)$ agreed to participate in the trial. After randomization $21(1.8 \%)$ women withdrew consent and $4(0.3 \%)$ were lost to follow-up. The progesterone and placebo groups were well balanced at baseline (Table 1).

In the progesterone group 15 women delivered before 24 weeks' gestation, including 2 pregnancy terminations (one for fetal abnormalities and another for social reasons); in the placebo group 26 women delivered before 24 weeks' gestation, including 3 pregnancy terminations (one for fetal abnormalities and two for social reasons). In the progesterone group 
26 women had indicated delivery at between $24^{+0}$ and $33^{+6}$ weeks' gestation, including 24 for preeclampsia and / or fetal growth restriction, one for abruption and one for obstetric cholestasis; in the placebo group there were 23 indicated deliveries, including 20 for preeclampsia and / or fetal growth restriction, one for abruption, one for severe maternal hydronephrosis and one for twin anemia-polycythemia sequence.

\section{Primary outcome}

After excluding births before 24 weeks and indicated deliveries before 34 weeks, spontaneous birth between $24^{+0}$ and $33^{+6}$ weeks of gestation occurred in $10.4 \%(56 / 541)$ participants in the progesterone group and in $8.2 \%(44 / 538)$ in the placebo group (odds ratio in the progesterone group, adjusting for the effect of participating center, chrorionicity, parity and method of conception, 1.35; 95\% Cl, 0.88-2.05; $\mathrm{p}=0.17$ ) (Table 2).

In the mixed effects logistic regression model for the primary analysis, the rate of spontaneous birth between $24^{+0}$ and $33^{+6}$ weeks was higher in women with monochorionic than dichorionic pregnancies (odds ratio 1.91; 95\% Cl 1.20-3.03; $p=0.006$ ), and in parous women with previous preterm births than in nulliparous women (odds ratio $2.631 ; 95 \% \mathrm{Cl} 1.26-5.48 ; \mathrm{p}=0.010$ ), but there was no significant difference between pregnancies conceived by in vitro fertilization and natural conception or those conceived after use of ovulation induction drugs (odds ratio 0.81; 95\% Cl 0.47-1.37; $p=0.43$ ) (Table S1 and S2 and Fig. S1 in the Supplementary Appendix).

Per-protocol analysis in the subgroup of women with adherence of $\geq 80 \%$ demonstrated that administration of progesterone, compared to placebo, was associated with a significant increase in the rate of spontaneous preterm birth between $24^{+0}$ and $33^{+6}$ weeks' gestation; $9.5 \%(42 / 443)$ participants in the progesterone group and 5.9\% (26/443) in the placebo group, (odds ratio in the progesterone group, adjusting for the effect of participating center, chrorionicity, parity and method of conception, $1.73 ; 95 \% \mathrm{Cl} 1.04$ to $2.91 ; p=0.037$ ). Nevertheless, the effect of vaginal progesterone on spontaneous birth between $24^{+0}$ and $33^{+6}$ weeks did not differ significantly between women with an adherence $\geq 80 \%$ and those with adherences of $60-79 \%$ and $<60 \%$ (Fig. S1 in the Supplementary Appendix).

For the intention to treat population, pre-specified tests showed no evidence of interaction between the effects of treatment and chorionicity $(p=0.28)$, parity $(p=0.35)$ method of conception $(p=0.56)$ and adherence $(p=0.34)$; however, there was weak evidence of an interaction with cervical length $(p=0.08)$ suggestive of harm to those with cervical length $\geq 30 \mathrm{~mm}$ (odds ratio $1.61,95 \% \mathrm{Cl} 1.01-2.59$ ) and potential benefit for those with cervical length $<30 \mathrm{~mm}$ (odds ratio 0.56; 95\% Cl 0.20-1.60) (Fig. S1 and S2 in the Supplementary Appendix).

\section{Secondary outcomes}

The treatment effect for secondary outcomes, quantified as odds ratio in the progesterone group with $95 \%$ confidence interval is shown in Table 2. There was no significant between-group difference in the incidence of any secondary outcomes. Interclass correlations for neonatal outcomes were calculated in Table S3 in the Supplementary Appendix.

\section{Time to event analyses}

The cumulative incidence of spontaneous birth between $24^{+0}$ and $33^{+6}$ weeks of gestation is shown in Fig. 2; the hazard ratio was $1.29(95 \% \mathrm{Cl} 0.87-1.91)$. Tests of interactions mirrored those from the logistic regression with no evidence of interaction between the effects of treatment and chorionicity, parity, method of conception or adherence. There was some evidence of an interaction between the effect of treatment and cervical length $p=0.049$ (Table S4 in the Supplementary Appendix). For those with cervical length $\geq 30 \mathrm{~mm}$ the hazard ratio was 1.58 (95\% $\mathrm{Cl} 1.01-2.47$ ) suggestive of harm, whereas for those with cervical length $<30 \mathrm{~mm}$ there was 
potential benefit (hazard ratio $0.49,95 \% \mathrm{Cl} 0.19-1.32$ ). There was also evidence of an interaction between the effect of treatment and cervical length for miscarriage or spontaneous birth from randomization to $33^{+6}$ weeks $(p=0.040)$, but not for all births between randomization and $33^{+6}$ weeks $(p=0.45)$ (Tables S5 and S6 in the Ssupplementary Appendix).

Cumulative incidences stratified by cervical length for spontaneous birth between $24^{+0}$ and $33^{+6}$ weeks, miscarriage or spontaneous birth between randomization and $33^{+6}$ weeks and all births between randomization and $33^{+6}$ weeks are shown in Fig. 3 . It is clear from the cumulative incidence curves that the proportional hazards assumption for the treatment effect is unrealistic because the cumulative incidence curves for progesterone and placebo crossed. This led to the estimation of hazard ratios stratified according to gestational age at birth shown in Fig. 4 and Table S7 in the Supplementary Appendix. The most notable feature of Fig. 4 is the relative reduction in risk (progesterone/placebo) of spontaneous births before $32^{+0}$ weeks in those with a cervical length less than $30 \mathrm{~mm}$. For all spontaneous births hazard ratio $0.23(95 \% \mathrm{Cl}: 0.08$ 0.69). This can be contrasted with an increase in the rate of spontaneous births between $32^{+0}$ and $33^{+6}$ weeks (hazard ratio $1.42,95 \% \mathrm{Cl}: 0.31-6.43$ ) which suggests that in those with short cervical lengths the effect of progesterone is to delay premature delivery; decreasing risks before 32 weeks but, increasing them between 32 and 36 weeks.

\section{Adverse events}

In the progesterone group there were $1.4 \%(8 / 582)$ women and $1.9 \%(22 / 1,164)$ fetuses with at least one serious adverse event; the respective numbers for the placebo group were $1.2 \%$ $(7 / 587)$ and $3.2 \%(37 / 1,174),(p=0.80$ and $p=0.06$, respectively) (Table 3$)$. In the progesterone group there were 200 (34.4\%) women with non-serious adverse events and the respective values in the placebo group were $186(31.7 \%),(p=0.35)$ (Table S8 in the Supplementary Appendix). There was no significant difference between the two groups in the incidence of preeclampsia, gestational hypertension, gestational diabetes mellitus and intrahepatic cholestasis (Table S9 in the Supplementary Appendix).

\section{Adherence}

Adherence was $\geq 80 \%$ in $952(81.4 \%)$ of the participants. There were no significant betweengroup differences in the degree of adherence (Table S10 in the Supplementary Appendix). A sensitivity analysis taking into account adherence to treatment is shown in Fig. $S 1$ in the Supplementary Appendix.

\section{COMMENT}

\section{Main findings of the study}

In this large multicenter randomized placebo-controlled trial involving women with twin pregnancies universal administration of progesterone at a dose of $300 \mathrm{mg}$ twice per day from 1114 until 34 weeks' gestation did not reduce the incidence of spontaneous birth between $24^{+0}$ and $33^{+6}$ weeks' gestation. There was no evidence of any difference between the groups in the incidence of other pregnancy complications, adverse fetal or neonatal outcomes, and maternal or fetal serious adverse events. Adherence to treatment was good with $>80 \%$ of the participants taking $\geq 80 \%$ of their capsules.

In pre-specified tests we found no evidence of interaction between the effects of treatment and chorionicity, parity, method of conception and adherence, but there was weak evidence of an interaction with cervical length; in the small subgroup of women with cervical length $<30 \mathrm{~mm}$ we could not exclude the possibility of benefit from vaginal progesterone. 
The selected primary outcome was spontaneous birth at $24^{+0}$ to $33^{+6}$ weeks' gestation, rather than all births between randomization and 34 weeks. We chose spontaneous rather than all preterm births because there is no reason to believe that progesterone would reduce indicated preterm births. As shown in Table 2 we found that the rates of all births at $24^{+0}$ to $33^{+6}$ weeks were $14.5 \%$ for the progesterone group and $11.9 \%$ for the placebo group. We excluded births before 24 weeks to allow comparison with the results of previous trials that recruited patients at mid-gestation. However, this exclusion could mask an effect of progesterone of converting late miscarriages to early preterm births; birth between randomization and 24 weeks occurred in $2.6 \%$ of pregnancies in the progesterone group and in $4.4 \%$ in the placebo group, but the rate of all births between randomization and 34 weeks were $16.7 \%$ for the progesterone group and $15.8 \%$ for the placebo group.

\section{Interpretation of results and comparison with findings of previous studies}

The findings of this study, where progesterone therapy was initiated in early pregnancy, are consistent with those of six previous smaller trials in unselected twin pregnancies that investigated the value of prophylactic use of lower doses of vaginal progesterone from midgestation and reported no significant effect on the incidence of early preterm birth..$^{8-13}$ Consequently, in twin pregnancies there is no benefit from universal prophylactic use of progesterone even when the dose is high and the drug is initiated from as early as 11 weeks' gestation. Indeed, our findings that the incidence of spontaneous early preterm birth is increased in women with good adherence to treatment and in those with cervical length $\geq 30 \mathrm{~mm}$ suggest that such treatment may actually be harmful. This observation has not been previously identified with a multiple gestation exposed to vaginal progesterone and raises the potential that harm may be related to the high dose, the early onset of therapy, or the duration of high dose therapy; however, further study is necessary. The identification of potential harm in this lowest risk subpopulation of women with a multiple gestation exposed to a different progestogen, 17-OHPC, has also been demonstrated by Schuit et al in an individual participant data meta-analysis. ${ }^{14}$ Therefore, providers should avoid use of any progestogen therapy in unselected twin pregnancies due to the accumulating evidence of potential harm.

Our finding of possible benefit from vaginal progesterone in the group with short cervix is consistent with the results of two individual participant data meta-analyses. One meta-analysis reported that although in women with unselected twin pregnancies vaginal progesterone from mid-gestation is not associated with reduction in the rate of preterm birth or adverse perinatal outcome, in a subgroup with short cervix progesterone reduces the rate of adverse perinatal outcome. ${ }^{14}$ Another individual participant data meta-analysis reported that administration of vaginal progesterone to asymptomatic women with a twin pregnancy and a mid-trimester sonographic short cervix significantly reduces the risk of both early preterm birth and neonatal death. ${ }^{22}$ Consequently, the small subgroup of women with short cervix merits further investigation.

Our finding of convergence of the cumulative incidence with gestational age together with the restricted beneficial effect of progesterone to births before 32 weeks' gestation suggests that the effect of progesterone is to delay the gestational age at delivery for those pregnancies destined to deliver before 32 weeks. This is similar to the suggested effect of aspirin in delaying deliveries due to preeclampsia. ${ }^{23}$

\section{Strengths and limitations}

Strengths of the study include its large size, high acceptance to randomization and adherence to treatment, and low rates of withdrawal and loss to follow up. However, the event rates were lower than anticipated so that the primary outcome was somewhat underpowered. Findings from the mixed effects Cox regression were the result of a post-hoc analyses and should be considered as exploratory. This is the first phase III study to suggest first trimester cervical length may be 
utilized to discriminate treatment response to vaginal progesterone and further study is required to validate this observation.

\section{Conclusions}

In conclusion, this randomized trial showed that in unselected twin pregnancies administration of progesterone at a dose of $600 \mathrm{mg}$ per day from 11-14 until 34 weeks' gestation did not reduce the incidence of spontaneous birth between $24^{+0}$ and $33^{+6}$ weeks' gestation. Post hoc time to event analysis led to the suggestion that progesterone may reduce the risk of spontaneous birth $<32$ weeks in -women with cervical length $<30 \mathrm{~mm}$ and it may increase the risk for those with cervical length $\geq 30 \mathrm{~mm}$. 


\section{Acknowledgements}

We thank all the participants and their attending obstetricians and midwives; Dr. George Attilakos, University College London Hospital, Dr. Bryony Jones, Imperial College Healthcare NHS Trust, Dr. Mark Turner, University of Liverpool, Dr. Christina Yu, Imperial College Healthcare NHS Trust, and lan Bradbury, Statistics at Frontier Science Scotland, all from the United Kingdom, and Dr. Anca Panaitescu, Filanthropia Hospital Bucharest from Romania for serving as members of the trial steering committee or independent data monitoring committee; the Clinical Trial Unit-IMIB Arrixaca-SCReN platform of Virgen de la Arrixaca University Clinical Hospital, Murcia in Spain (grant PI18/01027 Instituto Carlos III) for being responsible for the project management and oversight of the trial; and the following medical professionals who helped in the recruitment and follow-up of participants: Jessica Aguilera, Mercedes de Alvarado, Matthew Anderson, Brindusa Cimpoca, Hila de Castro, Mariana Catalano, Carla Coronel, Dejana Dojchinovska, Apostolos Georgantis, Andrea Harrington, Salma Jabak, Konstantinos Maligiannis, Alicia Mazer Zumaeta, Maria Mohova, Aikaterini Papanikolaou, Christina Resta, Angeliki Rouvali, Efsaia Savvoulidou, Maria Tsavdaridou, Theodora Tsokaki, Denisa Vojtassakova and Emilie Zingler from London, United Kingdom; Vangeliya Atanasova and Cristina Casanova, from Madrid, Spain; Juan Luis Delgado, Delia Lopez, and Macarena Quesada Rojas, from Murcia, Spain; Carol Diaz, and Tania De Ganzo, from Granada, Spain; Ana del Campo, from Barakaldo, Spain, Vivien Dutemeyer, from Brussels, Belgium; Veronica Accurti and IIma Floriana Carbone, from Milan, Italy; Vasil Kalev, from Sofia, Bulgaria; Fundación para la Formación e Investigación Sanitaria in Murcia, Spain represented by the Clinical Trials and Clinical Research Unit, acted as regulatory sponsor of this trial for all sites in Spain and the Fetal Medicine Foundation for sites in the UK, Bulgaria, Belgium, France and Italy. 


\section{REFERENCES}

1. United Nations Inter-agency Group for Child Mortality Estimation (UN IGME), 'Levels \& Trends in Child Mortality: Report 2019, Estimates developed by the United Nations Interagency Group for Child Mortality Estimation', United Nations Children's Fund, New York, 2019.

2. Martin JA, Osterman MJK. Describing the increase in preterm births in the United States, 2014-2016. NCHS Data Brief 2018;312:1-8.

3. To MS, Fonseca EB, Molina FS, Cacho AM, Nicolaides KH. Maternal characteristics and cervical length in the prediction of spontaneous early preterm delivery in twins. Am J Obstet Gynecol 2006;194:1360-5.

4. Fonseca EB, Celik E, Parra M, Singh M, Nicolaides KH: Fetal Medicine Foundation Second Trimester Screening Group. Progesterone and the risk of preterm birth among women with a short cervix. N Engl J Med 2007;357:462-9.

5. Hassan SS, Romero R, Vidyadhari D, et al. PREGNANT Trial: Vaginal progesterone reduces the rate of preterm birth in women with a sonographic short cervix: a multicenter, randomized, double-blind, placebo-controlled trial. Ultrasound Obstet Gynecol 2011;38:18-31.

6. Romero R, Conde-Agudelo A, Da Fonseca E, et al. Vaginal progesterone for preventing preterm birth and adverse perinatal outcomes in singleton gestations with a short cervix: a meta-analysis of individual patient data. Am J Obstet Gynecol 2018;218:161-80.

7. Likis FE, Edwards DR, Andrews JC, et al. Progestogens for preterm birth prevention: a systematic review and meta-analysis. Obstet Gynecol 2012;120:897-907.

8. Norman JE, Mackenzie F, Owen P, et al. Progesterone for the prevention of preterm birth in twin pregnancy (STOPPIT): a randomised, double blind, placebo-controlled study and metaanalysis. Lancet 2009;373:2034-40.

9. Rode L, Klein K, Nicolaides KH, Krampl-Bettelheim E, Tabor A, PREDICT Group. Prevention of preterm delivery in twin gestations (PREDICT): a multicenter, randomized, placebocontrolled trial on the effect of vaginal micronized progesterone. Ultrasound Obstet Gynecol 2011;38:272-80.

10. Wood S, Ross S, Tang S, Miller L, Sauve R, Brant R. Vaginal progesterone to prevent preterm birth in multiple pregnancy: A randomized controlled trial. J Perinat Med 2012;40:593-9.

11. Serra V, Perales A, Meseguer J, et al. Increased doses of vaginal progesterone for the prevention of preterm birth in twin pregnancies: a randomised controlled double-blind multicentre trial. BJOG 2013;120:50-7.

12. Brizot ML, Hernandez W, Liao AW, et al. Vaginal progesterone for the prevention of preterm birth in twin gestations: a randomized placebo controlled double-blind study. Am J Obstet Gynecol 2015;213:82.e1-9.

13. Shabaan OM, Hassanin IM, Makhlouf AM, Salem MN, Hussein M, Mohamed M, Abbas AM. Vaginal progesterone for prevention of preterm delivery in women with twin pregnancy: a randomized controlled trial. Facts Views Vis Obgyn 2018;10:93-98.

14. Schuit E, Stock S, Rode L, et al. Effectiveness of progestogens to improve perinatal outcome in twin pregnancies: an individual participant data meta-analysis. BJOG 2015;122:27-37. 
15. Greco E, Lange A, Ushakov F, Calvo JR, Nicolaides KH. Prediction of spontaneous preterm delivery from endocervical length at 11 to 13 weeks. Prenat Diagn 2011;31:84-9.

16. Nicolaides $\mathrm{KH}$, Wright $\mathrm{D}$, Syngelaki A, Wright $\mathrm{A}$, Akolekar R. Fetal Medicine Foundation fetal and neonatal population weight charts. Ultrasound Obstet Gynecol 2018;52:44-51.

17. $\mathrm{R}$ Development Core Team. R: A language and environment for statistical computing. $\mathrm{R}$ Foundation for Statistical Computing, Vienna, Austria. 2011 (http://www.R-project.org/).

18. Bates D, Maechler M, Bolker B, Walker S. Fitting linear mixed-effects models using Ime4. J Stat Soft 2015;67:1-48.

19. Therneau TM. A package for survival analysis in S. version 2.38, 2015 [cited 2020 Jan 6]; Available from: https://CRAN.R-project.org/package=survival

20. Therneau TM. Mixed effects cox models. R package version 2.2-14, 2019 [cited 2020 Jan 6]: Available from: https://CRAN.R-project.org/package=coxme

21. Revelle, WR, psych: Procedures for personality and psychological research, 2017. [cited 2020 Jan 6]: Available from: https://CRAN.R-project.org/package=psych

22. Romero R, Conde-Agudelo A, El-Refaie W, et al. Vaginal progesterone decreases preterm birth and neonatal morbidity and mortality in women with a twin gestation and a short cervix: an updated meta-analysis of individual patient data. Ultrasound Obstet Gynecol 2017;49:30314.

23. Wright $\mathrm{D}$, Nicolaides $\mathrm{KH}$. Aspirin delays the development of preeclampsia. Am J Obstet Gynecol 2019;220:580.e1-580.e6. 


\section{FIGURE LEGENDS}

Figure 1. Screening, randomization and follow-up.

Figure 2. Kaplan-Meier plot of cumulative percentage of participants who delivered spontaneously between $24^{+0}$ and $33^{+6}$ weeks' gestation.

Figure 3. Cumulative incidences stratified by cervical length for spontaneous birth between $24^{+0}$ and $33^{+6}$ weeks' gestation, miscarriage or spontaneous birth between randomization and $33^{+6}$ weeks and all births between randomization and $33^{+6}$ weeks. The blue curves are for the placebo group and the red ones for the progesterone group. The top three panels are for cervical length $<30 \mathrm{~mm}$ and the bottom three for cervical length $\geq 30 \mathrm{~mm}$.

Figure 4. Hazard ratios (progesterone / placebo) for spontaneous birth from $24^{+0}$ to $33^{+6}$ weeks' gestation, miscarriage of spontaneous birth between randomisation and $33^{+6}$ weeks, and all births between randomisation and $33^{+6}$ weeks by subgroup according to cervical length and by gestational age at delivery. 
Table 1. Characteristics of the trial participants.

\begin{tabular}{|l|c|c|}
\hline Characteristic & $\begin{array}{c}\text { Progesterone group } \\
(\mathbf{n}=582)\end{array}$ & $\begin{array}{c}\text { Placebo group } \\
(\mathbf{n}=587)\end{array}$ \\
\hline Gestation at randomization (weeks) & $13.2(12.7-13.6)$ & $12.2(12.7-13.7)$ \\
\hline Dichorionic pregnancies & $449(77.1)$ & $453(77.2)$ \\
\hline Monochorionic pregnancies & $133(22.9)$ & $134(22.8)$ \\
\hline Cervical length $(\mathrm{mm})$ & $34.4(31.0-38.0)$ & $34.6(31.5-38.0)$ \\
\hline Cervical length $<30 \mathrm{~mm}$ & $85(14.6)$ & $70(11.9)$ \\
\hline Age $($ years) & $34.1(30.3-37.7)$ & $34.0(30.0-37.6)$ \\
\hline Body mass index $\left(\mathrm{kg} / \mathrm{m}^{2}\right)$ & $24.7(21.9-28.4)$ & $24.3(22.0-27.9)$ \\
\hline Height $(\mathrm{cm})$ & $166(161-170)$ & $165(160-170)$ \\
\hline Weight $(\mathrm{kg})$ & $68.7(59.6-79.0)$ & $66.5(59.5-76.9)$ \\
\hline Race & & $492(83.8)$ \\
\hline White & $473(81.3)$ & $59(10.1)$ \\
\hline Black & $69(11.9)$ & $28(4.8)$ \\
\hline South Asian & $18(3.1)$ & $3(0.5)$ \\
\hline East Asian & $8(1.4)$ & $5(0.9)$ \\
\hline Mixed & $14(2.4)$ & $380(64.7)$ \\
\hline Conception & & $44(7.5)$ \\
\hline Natural & $382(65.6)$ & $163(27.8)$ \\
\hline Assisted by use of ovulation drugs & $35(6.0)$ & $39(6.6)$ \\
\hline In vitro fertilization & $165(28.4)$ & $7(1.2)$ \\
\hline Cigarette smoker & $36(6.2)$ & $3(0.5)$ \\
\hline Medical history & & $326(55.5)$ \\
\hline Chronic hypertension & $11(1.9)$ & $33(5.6)$ \\
\hline Diabetes mellitus type 1 or 2 & $8(1.4)$ & $228(38.8)$ \\
\hline Obstetrical history & & \\
\hline Nulliparous & $317(54.5)$ & $23(4.0)$ \\
\hline Parous with preterm birth $<37$ weeks & $242(41.6)$ & \\
\hline Parous without preterm birth $<37$ weeks & & \\
\hline
\end{tabular}

Data are presented as median $\left(25^{\text {th }}\right.$ to $75^{\text {th }}$ percentile) or $n(\%)$. 
Table 2. Outcomes according to trial group.

\begin{tabular}{|c|c|c|c|c|c|c|}
\hline \multirow{2}{*}{ Outcome measures } & \multicolumn{3}{|c|}{ Pregnancy level } & \multicolumn{3}{|c|}{ Neonatal/fetal level } \\
\hline & Progesterone group & Placebo group & OR $(95 \% \mathrm{Cl})$ & Progesterone group & Placebo group & OR $(95 \% \mathrm{Cl})$ \\
\hline \multicolumn{7}{|l|}{ Primary outcome } \\
\hline Spontaneous birth between $24^{+0}$ and $<34$ weeks & $56 / 541(10.4)$ & $44 / 538(8.2)$ & $1.35(0.88-2.05)$ & - & - & - \\
\hline \multicolumn{7}{|l|}{ Secondary outcomes } \\
\hline Any birth between randomization and $<24$ weeks & $15 / 582(2.6)$ & $26 / 587(4.4)$ & $0.57(0.30-1.10)$ & - & - & - \\
\hline Any birth between randomization and $<34$ weeks & $97 / 582(16.7)$ & $93 / 587(15.8)$ & $1.10(0.80-1.51)$ & - & - & - \\
\hline Any birth between $24^{+0}$ and $<34$ weeks & $82 / 567(14.5)$ & $67 / 561(11.9)$ & $1.28(0.90-1.82)$ & - & - & - \\
\hline Any birth between randomization and $<28$ weeks & $23 / 582(4.0)$ & $35 / 587(6.0)$ & $0.65(0.38-1.13)$ & - & - & - \\
\hline Any birth between randomization and $<30$ weeks & $31 / 582(5.3)$ & $49 / 587(8.3)$ & $0.63(0.39-1.01)$ & - & - & - \\
\hline Any birth between randomization and $<32$ weeks & $53 / 582(9.1)$ & $65 / 587(11.1)$ & $0.81(0.55-1.20)$ & - & - & - \\
\hline Any birth between randomization and $<37$ weeks & $330 / 582(56.7)$ & $322 / 587(54.9)$ & $1.13(0.88-1.46)$ & - & - & - \\
\hline Any birth between $24^{+0}$ and $<28$ weeks & $8 / 567(1.4)$ & $9 / 561(1.6)$ & $0.89(0.34-2.33)$ & - & - & - \\
\hline Any birth between $24^{+0}$ and $<30$ weeks & $16 / 567(2.8)$ & $23 / 561(4.1)$ & $0.70(0.36-1.34)$ & - & - & - \\
\hline Any birth between $24^{+0}$ and $<32$ weeks & $38 / 567(6.7)$ & $39 / 561(7.0)$ & $0.97(0.61-1.55)$ & - & - & - \\
\hline Any birth between $24^{+0}$ and $<37$ weeks & $315 / 567(55.6)$ & $296 / 561(52.8)$ & $1.16(0.90-1.51)$ & - & - & - \\
\hline Spontaneous birth between $24^{+0}$ and $<28$ weeks & $8 / 567(1.4)$ & $7 / 559(1.3)$ & $1.15(0.41-3.21)$ & - & - & - \\
\hline Spontaneous birth between $24^{+0}$ and $<30$ weeks & $14 / 565(2.5)$ & $16 / 554(2.9)$ & $0.88(0.43-1.84)$ & - & - & - \\
\hline Spontaneous birth between $24^{+0}$ and $<32$ weeks & $25 / 554(4.5)$ & $24 / 546(4.4)$ & $1.05(0.59-1.86)$ & - & - & - \\
\hline Spontaneous birth between $24^{+0}$ and $<37$ weeks & $161 / 413(39.0)$ & $137 / 402(34.1)$ & $1.36(1.00-1.83)$ & - & - & - \\
\hline Stillbirth or neonatal death & $12 / 582(2.1)$ & $9 / 587(1.5)$ & $1.41(0.58-3.39)$ & $15 / 1,164(1.3)$ & $10 / 1,174(0.9)$ & $1.57(0.70-3.53)$ \\
\hline Live births & $\mathrm{n}=569$ & $\mathrm{n}=565$ & & $\mathrm{n}=1,125$ & $n=1,113$ & \\
\hline \multicolumn{7}{|l|}{ Birth weight } \\
\hline$<1500 \mathrm{~g}$ & $51 / 569(9.0)$ & $50 / 565(8.8)$ & $1.03(0.68-1.56)$ & $75 / 1,125(6.7)$ & $76 / 1,113(6.8)$ & $0.93(0.65-1.32)$ \\
\hline$<2000 \mathrm{~g}$ & $174 / 569(30.6)$ & $159 / 565(28.1)$ & $1.16(0.89-1.50)$ & $259 / 1,125(23.0)$ & $239 / 1,113(21.5)$ & $1.13(0.92-1.40)$ \\
\hline$<3^{\text {rd }}$ percentile & $272 / 569(47.8)$ & $278 / 565(49.2)$ & $0.96(0.75-1.21)$ & $352 / 1,125(31.3)$ & $369 / 1,113$ (33.2) & $0.97(0.80-1.16)$ \\
\hline$<5^{\text {th }}$ percentile & $328 / 569(57.6)$ & $319 / 565(56.5)$ & $1.07(0.84-1.36)$ & $443 / 1,125(39.4)$ & $441 / 1,113(39.6)$ & $1.05(0.88-1.25)$ \\
\hline$<10^{\text {th }}$ percentile & $394 / 569(69.2)$ & $390 / 565(69.0)$ & $1.02(0.79-1.31)$ & $581 / 1,125(51.6)$ & $576 / 1,113(51.8)$ & $1.04(0.88-1.24)$ \\
\hline Neonatal therapy & $171 / 569(30.1)$ & $159 / 565(28.1)$ & $1.12(0.86-1.46)$ & $268 / 1,125(23.8)$ & $252 / 1,113(22.6)$ & $1.09(0.89-1.33)$ \\
\hline Admission to NICU & $157 / 569(27.6)$ & $148 / 565(26.2)$ & $1.09(0.83-1.43)$ & $242 / 1,125(21.5)$ & $227 / 1,113(20.4)$ & $1.09(0.89-1.34)$ \\
\hline Need for ventilation & $132 / 569(23.2)$ & $121 / 565(21.4)$ & $1.13(0.84-1.51)$ & $199 / 1,125(17.7)$ & $191 / 1,113(17.2)$ & $1.06(0.85-1.33)$ \\
\hline Neonatal morbidity & $56 / 569(9.8)$ & $57 / 565(10.1)$ & $1.00(0.67-1.48)$ & $77 / 1,125(6.8)$ & $85 / 1,113(7.6)$ & $0.90(0.65-1.25)$ \\
\hline Respiratory distress syndrome & $43 / 569(7.6)$ & $42 / 565(7.4)$ & $1.04(0.67-1.63)$ & $57 / 1,125(5.1)$ & $67 / 1,113(6.0)$ & $0.85(0.59-1.22)$ \\
\hline Intraventricular haemorrhage & $8 / 569(1.4)$ & $9 / 565(1.6)$ & $0.89(0.34-2.33)$ & $8 / 1,125(0.7)$ & $10 / 1,113(0.9)$ & $0.79(0.31-2.01)$ \\
\hline Anaemia & $20 / 569(3.5)$ & $23 / 565(4.1)$ & $0.87(0.47-1.61)$ & $25 / 1,125(2.2)$ & $29 / 1,113(2.6)$ & $0.85(0.49-1.46)$ \\
\hline Necrotising enterocolitis & $2 / 569(0.4)$ & $3 / 565(0.5)$ & $0.70(0.12-4.23)$ & $2 / 1,125(0.2)$ & $3 / 1,113(0.3)$ & $0.69(0.11-4.17)$ \\
\hline Retinopathy & $6 / 569(1.1)$ & $13 / 565(2.3)$ & $0.46(0.17-1.24)$ & $7 / 1,125(0.6)$ & $18 / 1,113(1.6)$ & $0.39(0.16-0.95)$ \\
\hline Sepsis & $15 / 569(2.6)$ & $16 / 565(2.8)$ & $0.95(0.46-1.96)$ & $17 / 1,125(1.5)$ & $18 / 1,113(1.6)$ & $0.95(0.49-1.86)$ \\
\hline
\end{tabular}

$\mathrm{OR}$ = odds ratio; $\mathrm{Cl}=$ confidence interval; $\mathrm{NICU}=$ neonatal intensive care unit 
Table 3. Serious adverse events among trial participants. *

\begin{tabular}{|c|c|c|}
\hline Serious adverse event & Progesterone group & Placebo group \\
\hline Number of mothers & 596 & 598 \\
\hline \multicolumn{3}{|l|}{ Maternal serious adverse events } \\
\hline Preeclampsia with prolonged hospital stay (5d) & 3 & 0 \\
\hline Eclampsia with prolonged hospital stay (10d) & 0 & 1 \\
\hline Pulmonary embolism with prolonged hospital stay (4d) & 1 & 0 \\
\hline Postnatal liver rupture with prolonged hospital stay (31d) & 1 & 0 \\
\hline Obstetric cholestasis with prolonged hospital stay $(2 d)$ & 0 & 1 \\
\hline Abnormal liver function tests & 1 & 1 \\
\hline Postpartum haemorrhage with 3 litre blood loss & 1 & 0 \\
\hline Gastritis with prolonged hospital stay (4d) & 0 & 1 \\
\hline Dyspnea with prolonged hospital stay (2d) & 1 & 0 \\
\hline Restrictive cardiomyopathy with prolonged hospital stay (4d) & 0 & 1 \\
\hline Urinary tract infection with prolonged hospital stay (3d) & 0 & 1 \\
\hline Maternal mirror syndrome in association with fetal hydrops & 0 & 1 \\
\hline Mothers with at least one serious adverse event ${ }^{\star \star}$ & $8 / 582(1.4)$ & $7 / 587(1.2)$ \\
\hline Number of fetuses & 1,192 & 1,196 \\
\hline \multicolumn{3}{|l|}{ Fetal serious adverse events } \\
\hline HIV transmission from the mother & 2 & 0 \\
\hline Trisomy 21 & 1 & 3 \\
\hline Agenesis of corpus callosum & 2 & 2 \\
\hline Rhomboencephalosynapsis & 0 & 1 \\
\hline Spina bifida & 0 & 1 \\
\hline Ventriculomegaly severe after death of donor in TTTS & 0 & 1 \\
\hline Ventriculomegaly severe and duplex kidneys & 1 & 0 \\
\hline Subependymal cyst & 1 & 0 \\
\hline Cleft lip and palate & 1 & 4 \\
\hline Hypoplastic left heart syndrome & 0 & 1 \\
\hline Dysplastic pulmonary valve & 0 & 1 \\
\hline Coarctation of the aorta & 0 & 2 \\
\hline Pulmonary artery stenosis & 0 & 1 \\
\hline Tetralogy of Fallot & 0 & 1 \\
\hline Right aortic arch & 0 & 1 \\
\hline Ventricular septal defect & 2 & 4 \\
\hline Oesophageal atresia & 2 & 0 \\
\hline Exomphalos bowel & 1 & 0 \\
\hline Inguinal bilateral hernia & 0 & 1 \\
\hline Malrotation of the intestine requiring surgery & 1 & 0 \\
\hline Anal atresia & 0 & 1 \\
\hline Renal agenesis unilateral & 1 & 0 \\
\hline Duplex kidneys & 1 & 0 \\
\hline Hypospadias & 1 & 6 \\
\hline Fibular hemimelia bilateral & 1 & 0 \\
\hline Polydactyly bilateral & 2 & 1 \\
\hline Talipes equinovarus bilateral or unilateral & 2 & 3 \\
\hline Hemivertebra & 0 & 1 \\
\hline Haemangioma & 1 & 0 \\
\hline Sacrococcygeal teratoma & 2 & 0 \\
\hline Fetuses with at least one serious adverse event ${ }^{\star \star}$ & $22 / 1,164(1.9)$ & $37 / 1,174(3.2)$ \\
\hline
\end{tabular}

Data are presented as $\mathrm{n}$ or $\mathrm{n}(\%)$.

* None of these serious adverse events was considered by the investigators to be associated with progesterone or placebo.

** Women who withdrew their consent for participation in the study did not allow their data collected prior to withdrawal to be used in any analysis. 